\title{
Increasing Prevalence of Osteoporosis, Hypothyroidism and Endogenous Estrogen with Germ Cells
}

\author{
Elizabeth Jeya Vardhini Samuel, Nagarajan Natarajan, Sivakumar Periasamy, \\ Joseph Vimal, Sanjoy George, Gouthaman Thiruvenkadam \\ Department of General Medicine, Pondicherry Institute of Medical Sciences, Pondicherry, India \\ Email: elizabethjsamuel@gmail.com, vidya.nagarajan52@gmail.com, ep.sivadr@gmail.com, \\ josephvimal96@gmail.com, sanjoygeorge@hotmail.com, goutham.thiru.gt@gmail.com
}

Received 1 May 2014; revised 1 June 2014; accepted 1 July 2014

Copyright (C) 2014 by authors and Scientific Research Publishing Inc.

This work is licensed under the Creative Commons Attribution International License (CC BY). http://creativecommons.org/licenses/by/4.0/

c) (i) Open Access

\begin{abstract}
Background: Women were expressing disabling low back aches and weight gain attributing to puerperal sterilization, when contraception implementation as Family Welfare Program was progressing between $\sim 1983$ and $\sim 1989$. Hence we sought to identify any correlation existing with contraception. Methods: We performed a retrospective analysis of prevalence of osteoporosis and hypothyroid status, in 350 patients of 20 - 35 years, 35 - 50 years, > 50 years age groups, from data collected by convenient, stratified random sampling of different geographical locations, between 1995-2012. We also analyzed its association with presence or absence of contraception and abortion. Simultaneously, serum estrogen levels obtained from 105 patients were also analyzed. Results: $\sim 15$ - 25 fold increase in osteoporosis was seen in contraceptive users with a $p$ value of $<0.0005$ among $>20$ - >50 years age group; we also found a 6 fold increase in hypothyroidism among contraceptive users aged $>35$ years [p $<0.0005$ ], 3 fold increase in hypothyroidism among $>20$ years age group [p $<0.02$ ] and 1.5 fold increase in $>50$ years age group [p $<0.025]$. Endogenous estrogen was reduced in $61 \%$ of people using contraception with a $p$ value of $<0.0005$. Conclusion: Concept is that contraception prevents traversal of normal path by germ cells, resulting in smashed fragmentation of germ cells, and consequent decreased endogenous estrogen or androgen leading to defaulted genomic repertoire, deranged cell cycle and cell metabolism resulting in metabolic syndrome, with consequent increase in osteoporosis and hypothyroid status.
\end{abstract}

\section{Keywords}

Contraception Reversal, Smashed Fragmentation of Germ Cells, Reduced Endogenous Estrogen, Germ Cells Replant Effect 


\section{Introduction}

Since 1983 contraception [puerperal sterilization mostly] had got stealthily implemented in the name of Family Welfare program, without evidence for safety or therapeutic indication, i.e., there were no studies on pre or post implementation; young women were experiencing disabling low back aches, weight gain and were correlating these with sterilization; whereas medical fraternity attributed no significance to this, since the procedures were uneventful and the medical curriculum claimed no ill effects [for condoms, copper-t, vasectomy and tubectomy]; clinical help sought by the patients for their low back aches and obesity at young age led to this analysis to seek any altruistic relationship as the cause and effect phenomenon.

\section{Methods}

As advised by a scientist, 30 samples being essential for statistical analysis, minimum of 30 samples was planned for, in each of 3 age groups, namely 20 - 35 years, 36 - 50 years, >50 years; though people from the community were visiting the hospital, analysis of hospital patients alone could create a bias, hence data from the community, hospital, health screening camps, of different geographical locations were included; data from each person depicted, prevalent diseases including osteoporosis, hypothyroidism, status of contraception, hysterectomy, type of oil ingested, life style, level of nutrition, and presence of anemia; the data was tabulated as prevalent diseases, matched against the variables in each age group; in 2012 retrospective bioinformatics analysis was done, by plotting histograms for the 3 age groups and cumulative graphs for each disease including osteoporosis and hypothyroid disease; an example of tabulation of the data is provided in the supplementary file.

In 2002 house to house survey in the community, spread over 3 weeks, was conducted by the corresponding author, to collect data of prevalent diseases of 100 people; the people who were present during the survey were included at random, by convenient sampling into the 3 age groups namely 20 - 35 years, 35 - 50 years, >50 years, to include a minimum of 30 people in each age group; serum estrogen estimation was done for 12 people as per their request; the reduced estrogen levels [5 - 8 pg] found in young contraceptive users, was the eye opener, leading to further data analysis.

In 2004 data of 93 hospital patients were collected over a period of 6 months, including diseases prevalent, contraception status, life style, nutrition, type of oil ingested, level of hemoglobin and were assigned to the 3 age groups by stratified random sampling with a minimum of 30 patients in each age group; serum estrogen estimation was done for all 93 patients; the data was tabulated matching diseases against status of contraception and other variables; one patient was a foreign national.

In 2011, 96 people [43 couples] working in different states of our nation had attended a health screening camp conducted in the community, spread over 3 days and their data was analyzed after assigning into the 3 age groups at random, for association of diseases, including osteoporosis, hypothyroidism with status of contraception, hysterectomy and other variables; effect of contraception in both partners after contraception also could be analyzed; none had sedentary life style, low nutrition or anemia or had worn tight attires around the pelvis.

In 2012, data of 61 hospital patients including a foreign resident, from another geographical location, were collected over a span of 6 months, assigned to the 3 age groups at random and were pooled to the other data from 2003, 2004, 2011 and retrospective bio informatics analysis was undertaken for the 350 patients, by plotting histogram for the 3 age groups and cumulative graphs for each disease including osteoporosis and hypothyroid status.

Every participant was informed about their data being included for study purpose and the concerned hospital authorities were also informed; an engineering college student did the bio informatics analysis as his project.

\section{Results}

15 - 25 fold increase in osteoporosis among $>20$ - >50 years with a p value of $<0.0005$ as manifested by low bone mineral density and low back aches in people using contraception was observed Figure 1 [2002-2012]; estrogen levels were low in $61 \%$ of people using contraception with a p value of $<0.0005$ Figure 2 ; in $25 \%$ of people on contraception estrogen levels were low normal; but after hysterectomy alarmingly low levels as that of $0.4 \mathrm{pg}$ were seen (path of no return i.e., contraception reversal is not possible).

Weight gain with low thyroid function was seen among contraceptive users 6 fold more among 35 years age group with a p value of $<0.0005$; 3 fold increase among people using contraception $>20$ years with a $\mathrm{p}$ value of 


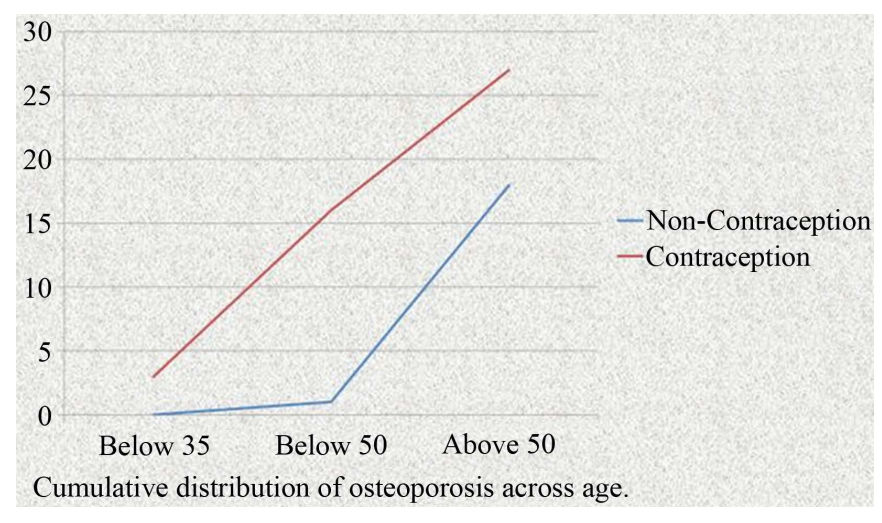

Figure 1. Cumulative distribution of osteoporosis and contraception.

$<0.02$; 1.5 fold increase was observed in people using contraception among $>50$ years age group with a p value of $<0.025$ Figure 3 ; with $2 \%$ of them having autoimmune mechanism documented by fine needle aspiration cytology, antibodies to thyroid peroxidase and thyroglobulin.

Cholesterol deprived diet i.e., oil without cholesterol \{[refined, bleached oils] sunflower seed has 6 times less vitamin $\mathrm{E}$ than ground nut seed, so sunflower refined oil\} resulted in $50 \%$ increase in osteoporosis, hypothyroid status in young people without contraception.

Contraception reversal reverted osteoporosis, hypothyroid status, by $79.9 \%$ with return of estrogen, halt of germ cell destruction-Figure 4.

\section{Discussion}

Osteoporosis is characterized by decreased bone strength, prevalent among post menopausal women [1] but also occurs in other men and women; common manifestations are hip, vertebral fractures; >10 million people have osteoporosis in United States of America but only a small proportion is diagnosed and treated.

Loss of bone tissue is associated with deterioration in skeletal micro architecture; World Health Organization defines osteoporosis as bone density that falls $2.5 \mathrm{SD}$ below the mean for young healthy adults of the same sex referred to as T-score of -2.5; post menopausal women who fall at the lower end of normal range as $\mathrm{T}$ score < -1.0 are defined as having low bone density and are also at increased risk of osteoporosis, more than $50 \%$ fractures among post menopausal women including hip fractures occur in this group with low bone mineral density.

In United States of America, 8 million women, 2 million men have osteoporosis [T score $<-2.5$ ] and additional 18 million have bone mass levels with increased risk of developing osteoporosis [bone mass $\mathrm{T}$ score $<$ $-1.0]$; as age increases loss of bone tissue increases progressively.

1.5 billion fractures in United States are due to osteoporosis, 30,000 hip fractures in 1 year , producing immediate mortality of $20 \%-50 \%$, due to associated deep vein thrombosis, pulmonary embolism; risks for blacks is reduced by $1 / 2$.

Pathology refers to bone loss due to age related changes in bone remodeling as well as extrinsic and intrinsic factors that exaggerate this process; these changes are superimposed on low peak bone mass; skeleton increases in linear growth and by apposition of new bone tissue on the outer surfaces of cortex, the latter process is called modeling a process that allows the bones to adapt in shape to the stresses placed on them;

Increased sex hormones production at puberty is required for skeletal maturation which reaches maximum mass and density in early adulthood; its around puberty sexual dimorphism [i.e. differences in shoulder girdle for males, gynecoid pelvis in females] in skeletal size becomes obvious; nutrition plays an important role in growth.

Numerous genes control skeletal growth, peak bone mass, body size as well as skeletal structure and density; association studies of candidate genes for vitamin D receptor, collagen type 1, estrogen receptor, interleukin b, insulin like growth factor-1, bone mass and bone turnover have been inconsistent; lipoprotein receptor related protein-gene signaling appears to be important in controlling bone formation.

Bone remodeling is the principal metabolic skeletal process; bone remodeling has two primary functions; to repair micro damage within skeleton to maintain skeletal strength and to supply calcium from the bone to maintain serum calcium. 


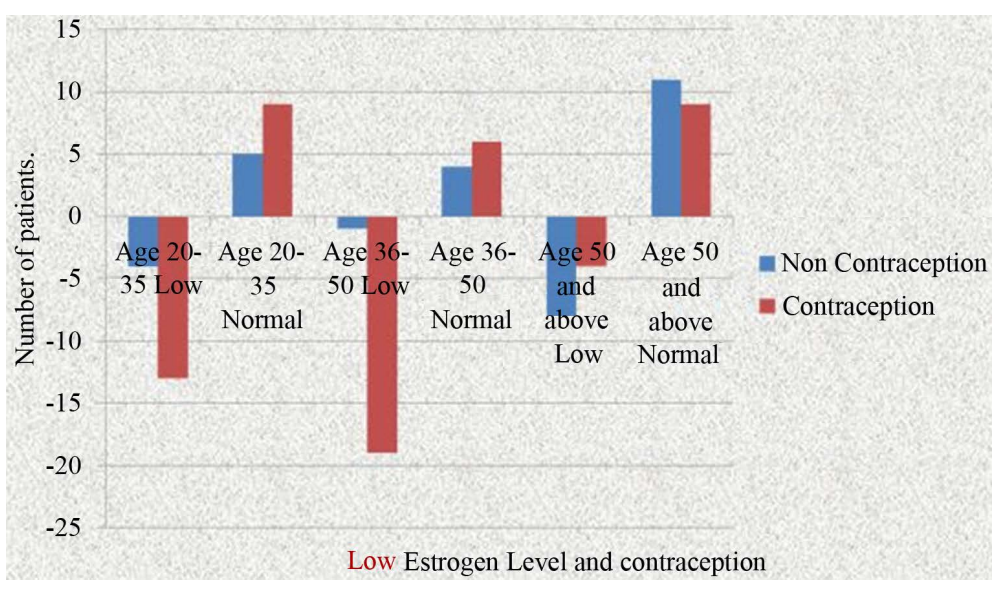

Figure 2. Reduced endogenous estrogen levels, contraception.

Cytokine responsible for communication between osteoblasts and other marrow cells and osteoclasts are identified as RANK ligand [receptor activator of nuclear factor kappa B] member of tumor necrosis factor, secreted by osteoblasts; a humoral decoy for RANKL secreted by osteoblasts is referred to as osteoprotearin

Bone remodeling is regulated by several circulating hormones including estrogens, androgens, vitamin D, Parathyroid Hormone, as well as locally produced growth factors such as IGF-1, immune reactive growth hormone-2, transforming growth factor $\beta$, interleukin, prostaglandins, and PTH related peptides.

In young adult the amount of bone resorbed is replaced by new bone tissue; mass of skeleton remains constant after peak bone mass is achieved; >30 - 45 years more bone resorption than new bone formation- this imbalance occurs at different ages.

$1000 \mathrm{mg}-1200 \mathrm{mg}$ of minimal calcium intake is required; vitamin D insufficiency occurring in liver, kidney diseases, in northern poles is detrimental to the appropriate homeostatic mechanisms for maintaining calcium economy.

In United Kingdom fracture related to osteoporosis accounts for $30 \%$ in females, $12 \%$ in males is a major public health concern; annually 250,000 individuals are treated for fractures; treatment of hip fractures associated with osteoporosis forms majority of health costs $\sim 1.75$ billion [2].

Endogenous estrogen deficiency causes uncoupling of bone resorption and new bone formation such that bone removed by osteoclasts exceeds the rate of new bone formation by osteoblasts; stem cells of bone marrow do not differentiate into osteoblasts but differentiate into adipocytes [degenerative change]; smoking, hypogonadism, corticosteroid use, and alcoholism are detrimental to bone mineral density.

Post pubertal females, an orderly secretion of gonadotropins [3] (p. 282) is necessary for the occurrence of menstruation, pregnancy, lactation.

Endogenous Estrogen prevents osteoporosis probably by a direct effect on osteoblasts [3] (p. 348, 371) exogenous estrogen achieves contraception, osteoporosis, by suppressing endogenous estrogen.

Vitamin D-closely related sterols produced by the action of ultra violet sunlight on certain provitamins in the skin is converted to 25 hydroxy cholecalciferol in the liver which further gets converted to 1,25 dihydroxy cholecalciferol - the more active metabolite in the proximal tubules of kidney and is also called as calcitriol; also made in placenta, macrophages, and keratinocytes of skin; vitamin D3 and its derivatives are seco-steroids

Estrogen is required for the cells to go through differentiation followed by controlled multiplication to become tissue and the genomic repertoire i.e., embryo like healing requires estrogen surveillance [4].

The concept is contraception [condoms, copper t, tubectomy, vasectomy] by its smashed, agonizing fragmentation of germ cells [>20 million sperms/day, many oocytes/mid menstrual cycle] to acentric fragments, ring chromosomes, chromatid breaks [5] and associated reduced levels of estrogen-[Figure 3] mediated through endorphins probably, to hypothalamic pituitary axis, [but presumed to be with no side effects and talked of as achievement to control population-blessed human race, as per guillotine protocol, without any therapeutic indication as against the principles and practice of medicine] results in increased prevalence of osteoporosis posing a major global health concern.

Since endogenous estrogen is reduced after global contraception implementation, osteoporosis has increased globally. 


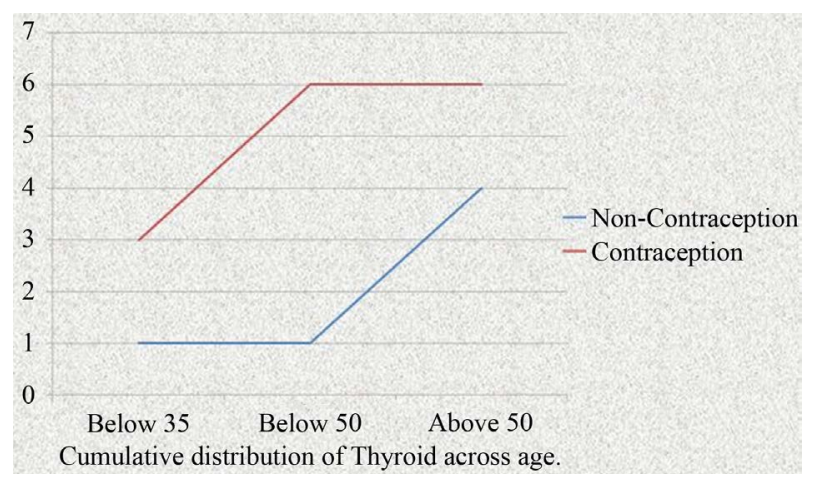

Figure 3. Cumulative distribution of thyroid diseases and contraception

Cholesterol or low density, intermediate, high density lipoproteins obtained from oil derived from nuts, seeds containing seedlings, growth, life factors including phytoestrogen, vitamin E, essential fatty acids, gets converted to our androgen or estrogen-steroid hormones the name itself being derived from chole-sterol [6] [7]; hence cholesterol deprived diet is associated with $50 \%$ increase in osteoporosis; olive oil has the highest fertility index; bleached, refined oil consumption, sunflower seed having 6 times less vitamin $\mathrm{E}$ than ground nut seed, so sunflower refined oil consumption results in osteoporosis; combined palm olein oil, gingili oil, ground nut oil, coconut oil, vegetable oil with its cholesterol consumption, reduces osteoporosis $50 \%$.

T4, T3 are synthesized in the colloid by iodination and condensation of tyrosine molecules bound in peptide linkage in thyroglobulin. The glycoprotein is synthesized in the thyroid cells secreted into the colloid by exocytosis of granules and contain thyroid peroxidase; hormones are secreted, colloid is ingested by thyroid cells; peptide bonds are hydrolyzed, free T3, T4 are discharged into the [8] (p. 306) capillaries; thyroid is estrogen dependent as any other cell function is.

A subunit in human chorionic gonadotrophin is the same as that in thyroid stimulating hormone; large amounts of human chorionic gonadotrophin can activate thyroid receptors [8] (p. 307).

Thyroid hormones potentiate the effects of growth hormone; growth hormone secretion is depressed in the absence of thyroid hormone;

Although sub acute thyroiditis, De Quervains thyroiditis, or viral thyroiditis is associated with Coxsackie virus, mumps, influenza, adenovirus, echo virus; causation has not been proven; patchy inflammatory infiltrate, disruption of thyroid follicles, multi nucleated giant cells are seen in some follicles [9] (pp. 2913-2914); 250 $\mu \mathrm{gm}$ of levo thyroxin is required in pregnancy and lactation.

Antibodies to thyroid peroxidase and thyroglobulin are markers of auto immunity; uniform enlargement of thyroid is typical for Hashimotos thyroiditis [9] (pp. 2917-2918); auto immune disease at the later stages have minimal residual thyroid tissue-atrophic thyroiditis

Thyroid diseases predominantly affect females, common in $5 \%$ of population; thyroid axis is involved in regulation of cellular differentiation [10] metabolism of all nucleated cells so that disorders of thyroid function have diverse manifestations; over supply of iodine by shell fish source is associated with auto immune thyroiditis.

The Concept is that consumption of shell fish or fish without scales and gills can result in urticaria and angio neurotic edema, either by molecular mimicry or toxin-hapten mediated mechanisms being associated with varieties of auto immune diseases including thyroiditis and nephritis.

Contraception with its associated reduced estrogen, fragmentation of germ cells results in hypothyroidism secondary to degenerative changes because estrogen surveillance dependent genomic repertoire defaults; thyroid function is estrogen dependent; auto immune mediation, i.e., fragmented abnormal nuclear materials of germ cells are recognized as foreign, leading to varied auto immunity including thyroiditis; estrogen, growth hormone and thyroid hormones with inter dependency when reduced after contraception lead to obesity and weight gain without secondary sexual character build or sexual dimorphism.

Contraception reversal as a cause and effect phenomenon reduces osteoporosis, hypothyroid status by $79.9 \%$ by halt of germ cells destruction and regeneration of endogenous estrogen [color-Figure 4-supplementary file]. 


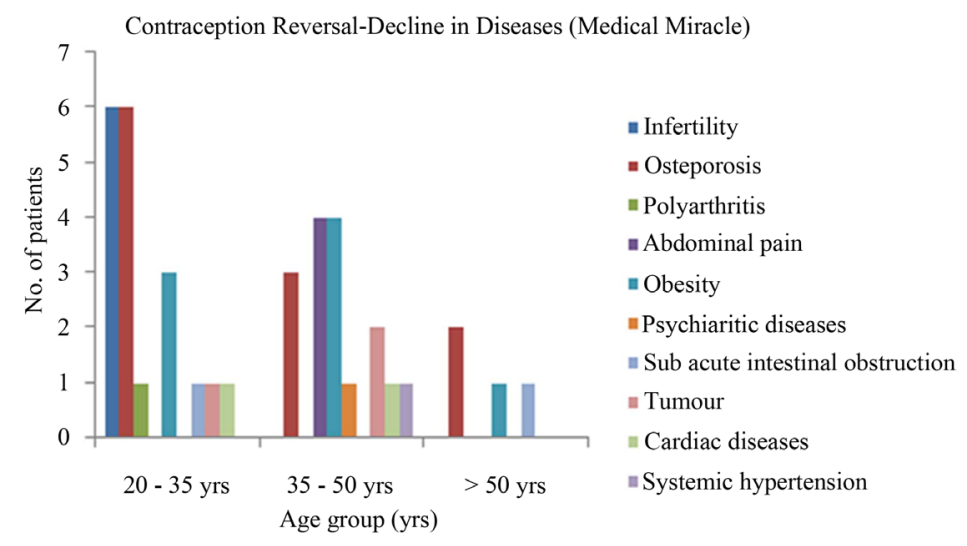

Figure 4. Contraception reversal, medical miracle decline in diseases.

\section{Conclusions}

Osteoporosis with its fractures forms a major health concern; thyroid disorders are common affecting $5 \%$ of population; contraception is associated with 15 - 25 fold increase in osteoporosis with a p value of $<0.0005$.

Contraception is associated with 6 fold increase in hypothyroid status among $>35$ years age group [p < 0.0005 ]; 3 fold increase among $>20$ years age group [p $<0.02$ ]; 1.5 fold increase among $>50$ years age group [p $<0.025]$. Estrogen levels are reduced in $61 \%$ of contraceptive users [p $<0.0005]$ and low normal range are seen in $25 \%$ of contraceptive users.

The concept is contraception with its associated smashed fragmentation of germ cells, reduced estrogen: androgen accounts for the increased osteoporosis by defaulted remodeling-skeletal metabolism, hypothyroid status by degenerative changes mainly and auto immune mediation [in a smaller percentage - $2 \%$ thyroiditis].

Cholesterol deprived diet [consumption of oil without fatty acids, i.e., refined, bleached oils and sunflower oil] due to decreased endogenous estrogen synthesis also increases osteoporosis by $50 \%$.

Shell fish: fish without scales and gills leads to thyroiditis probably by molecular mimicry or hapten-toxin mediated auto immunity.

Contraception with smashed abnormal fragmented nuclear materials of germ cells being recognized as foreign results in varieties of auto immune spectrum including thyroiditis [specially by exposure of fragmented chromatids to raw endometrial surface during 7 days after last menstrual period, or exposure of fragmented germ cells to raw detached placental surface in $<45$ days after male child birth, and $<90$ days after female child birth]; in people without contraception, exposure of germ cells to raw endometrial surface and detached placental surface can result in generation of autoimmunity but profound auto immune destruction including thyroiditis that is seen in people using contraception.

Contraception reversal decreases osteoporosis and hypothyroid status, as medical miracle by reversal of germ cell destruction and restoration of endogenous estrogen by $79.9 \%$.

\section{Key Points}

- Contraception with abnormal, acquired fragmentation of germ cells and reduced endogenous estrogen has resulted in 25 fold increase in osteoporosis among > 20 - >50 years age group with a p value of $<0.0005$;

- Contraception with reduced endogenous estrogen, resulting in degenerative defaulted genomic repertoire, leads to an increase in hypothyroidism by 6 fold [p $<0.0005$ ] among $>35$ years age group;

- Contraception: abortion and consumption of shell fish: fish without scales and gills can lead to auto immune thyroiditis;

- Cholesterol deprived diet with decreased estrogen synthesis leads to 50\% increase in osteoporosis and hypothyroidism;

- Contraception reversal decreases osteoporosis and hypothyroid status as autologous germ cell replant effect with restored endogenous estrogen.

\section{Conflicts of Interests}

None Declared. 


\section{Acknowledgements}

Mr. Jobin John—Final Year Bioinformatics student helped in analysis.

\section{References}

[1] Lindsay, R. and Cosman, F. (2012) Osteoporosis. In: Longo, D.L., Kasper, D.L., Jameson, J.L., Fauci, A.S., Hauser, S.L. and loscalzo, J., Eds., Harrison's Principles of Internal Medicine, McGraw Hill Medical, New York, Volume 2, 3120-3123.

[2] Doherty, M. and Ralston, S.H. (2010) Osteoporosis. In: Colledge, N.R., Walker, B.R. and Ralston, S.H., Eds., Davidson's Principles and Practice of Medicine. Churchill Livingstone Elsevier, Britain, 1116.

[3] Ganong, W.F. (2010) The Gonads: Review of Medical Physiology. Tata McGraw Hill, New Delhi, 348,371.

[4] Sohn, J.M. (1987) Principles of Neoplasia. Tumor Cell Biology. In: Baunwald, E., Isselbacher, K.J., Petersdorf, R.G., Wilson, J.D., Martin, J.B. and Fauci, A.S., Eds., Harrison’s Principles of Internal Medicine, McGraw Hill Book Company, New York, Volume 1, 422-423.

[5] Bruce, G. (1987) Gilliland: Systemic Sclerosis. In: Baunwald, E., Isselbacher, K.J., Petersdorf, R.G., Wilson, J.D., Martin, J.B. and Fauci, A.S., Eds., Harrison's Principles of Internal Medicine, McGraw Hill, New York, Volume 2, 1429.

[6] Brown, M.S. and Joseph, L. (1985) Goldstein: Exogenous, Endogenous Path Way. In: Gilman, A.G., Goodman, L.S., Rall, T.W. and Murad, F., Eds., Goodman and Gilman's The Pharmacological Basis of Therapeutics, Macmillan Publishing Company, New York, 827-828.

[7] Carr, B.R. and Wilson, J.D. (1987) Disorders of the Ovary and Female Reproductive Tract. In: Baunwald, E., Isselbacher, K.J., Petersdorf, R.G., Wilson, J.D., Martin, J.B. and Fauci, A.S., Eds., Harrison's Principles of Internal Medicine, McGraw Hill, New York, Volume 2, 1820-1821.

[8] Ganong, W.F. (2010) Thyroid. Review of Medical Physiology, Tata McGraw Hill, New Delhi, 306,307.

[9] Jameson, J.L. and Weetman, A.P. (2012) Disorders of the Thyroid Gland. In: Longo, D.L., Kasper, D.L., Jameson, J.L., Fauci, A.S., Hauser, S.L. and loscalzo, J., Eds., Harrison's Principles of Internal Medicine, McGraw Hill, New York, Volume 2, 2913,2914,2917,2918.

[10] Strachan, M.W.J. and Walker, B.R. (2010) Endocrine Disease. In: Colledge, N.R., Walker, B.R. and Ralston, S.H., Eds., Davidson's Principles and Practice of Medicine, Elsevier Science Ltd., Britain, 736. 
Scientific Research Publishing (SCIRP) is one of the largest Open Access journal publishers. It is currently publishing more than 200 open access, online, peer-reviewed journals covering a wide range of academic disciplines. SCIRP serves the worldwide academic communities and contributes to the progress and application of science with its publication.

Other selected journals from SCIRP are listed as below. Submit your manuscript to us via either submit@scirp.org or Online Submission Portal.
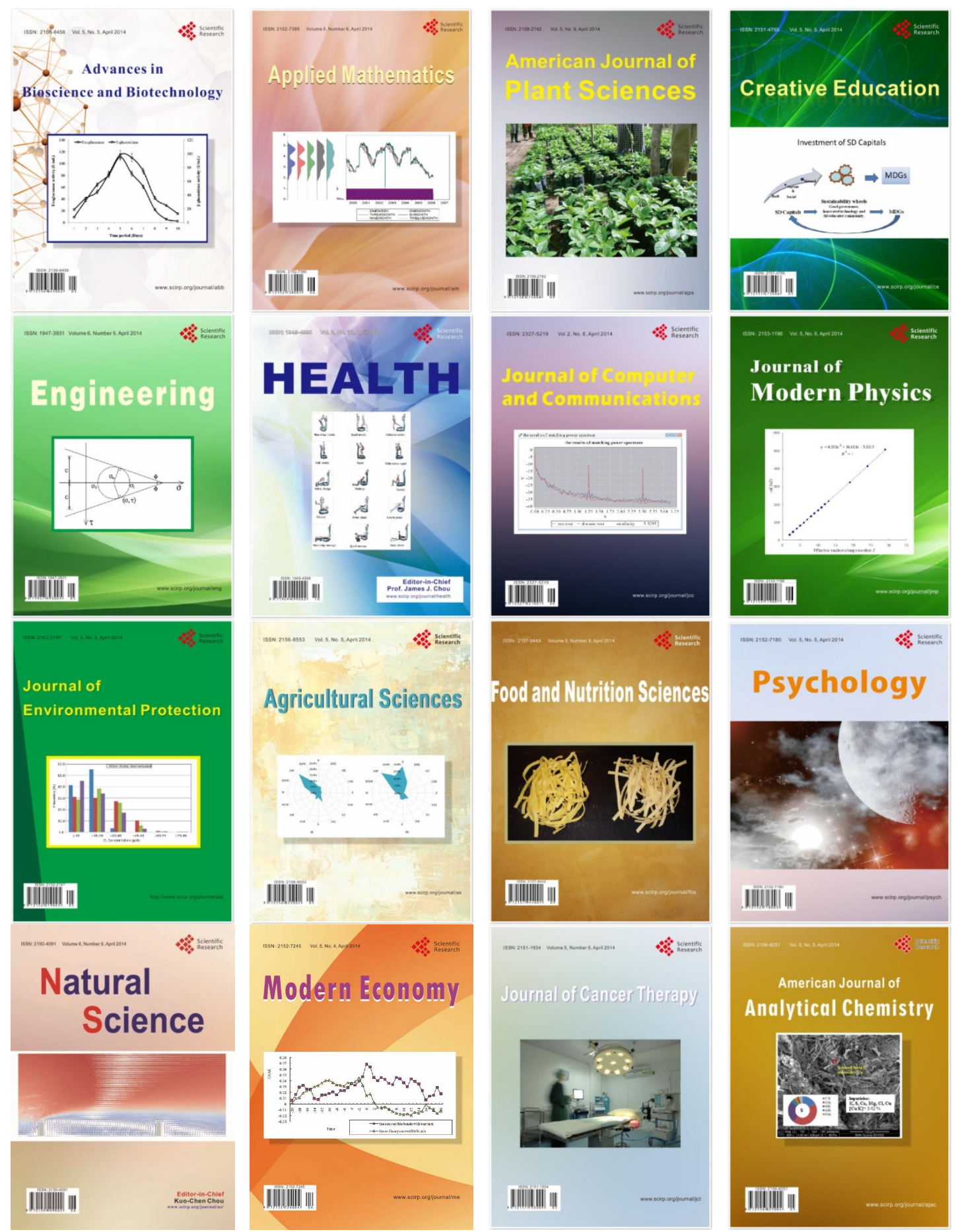